\title{
Effective Mass Model Reduced to Ordinary Mass Using Newton's, Quantum and Generalized Special Relativity
}

\author{
Nagwa Idriss Ali Ahmed1,2, Mohamed Idriss Ahmed3', Mubarak Dirar Abd-Alla ${ }^{3}$ \\ ${ }^{1}$ Department of Physics, Faculty of Science and Art (Dariah), Qassim University, Almulid, KSA; ${ }^{2}$ Department of \\ Physics and Mathematics, Hantoub Faculty of Education, Gezira University, Hantoub, Sudan; ${ }^{3}$ Department of \\ Physics, Faculty of Science, Sudan University of Science and Technology, Khartoum, Sudan
}

Correspondence to: Mohamed Idriss Ali Ahmed, garaof@yahoo.com

Keywords: Effective Mass, Newton's Second, Quantum Momentum, Generalized Special Relativity

Received: July 13, $2021 \quad$ Accepted: September 7, $2021 \quad$ Published: September 10, 2021

Copyright $\odot 2021$ by author(s) and Scientific Research Publishing Inc.

This work is licensed under the Creative Commons Attribution International License (CC BY 4.0).

http://creativecommons.org/licenses/by/4.0/

\section{(c) (i) Open Access}

\section{ABSTRACT}

The concept of the effective mass in crystals shows that the electron mass is affected by the crystal field and was experimentally verified. A useful expression for effective mass was obtained. Unfortunately this expression showed that the effective mass vanishes in the absence of the external field. This is in conflict with observations which show that it reduces to the ordinary mass. To cure this defect a new model is developed assuming the existence of vacuum force as verified experimentally as shown by Casimir effect. Using Newton's second law and the quantum expression of momentum, useful expressions were found. The same expression was found using generalized special relativity. Strikingly the three models reduced to the conventional one in the absence of vacuum, they also reduced to the ordinary electron mass in the absence of all forces.

\section{INTRODUCTION}

Material science is one of the most important branches of physics. It is concerned with the physical properties of matter to be utilized in modern technology [1]. This leads to trying to explain the behavior of matter by constructing theoretical models. These models concentrate mainly on knowing the factors that control the behavior of matter [2]. For instance researchers study how mechanical, thermal treatments, in addition to the doping process can control matter properties. These include magnetic, electric, thermal and mechanical properties [3].

The control of physical properties of matter helps too much in designing useful devices for human life. One of the most widely used properties is the electrical properties of bulk matter. The control of these properties is now widely used in designing electronic devices like computers, mobile telephones, televisions and controlling systems [4].

The behavior of the electrons in the electronic materials mainly determines how to control the elec- 
tronic properties of the bulk matter. These properties include electron mobility, diffusion, electric and magnetic interaction, besides the electron response to thermal energy and collision process [5]. These properties of the electron depend mainly on the electron mass and charge. However the mass seems to be more effective, since the mass effects determine the electron response to thermal, mechanical and electrical interactions, whereas the charge responds only to the electrical properties [6].

This important role played by the mass, encourages scientists to search for the factors that affect the electron mass [7]. One of the most important theories that changes our vision about mass is the special relativity (sr) proposed by Einstein [8].

According to sr the mass is affected by its speed, where it increases with the speed [9]. Recently the so called generalized sr (gsr) or potential dependent sr (psr) was proposed by Mubarak Dirar [10]. He showed that the mass is affected by the potential as well as the speed [11]. The main physical phenomena which confirm his belief are the gravitational red shift and the effective mass phenomenon. In the gravitational red shift phenomenon the photon mass increases due to the gravitational potential [12]. While in the electron effective mass phenomenon the electron mass is affected by the crystal field [13].

The model proposed to explain the effective mass phenomenon agrees with experimental observations to a great extent and higher degree of precession. However it suffers mainly from the fact that in the absence of the external force the effective mass vanishes. This is in direct conflict with observations, where the effective mass reduces to the ordinary mass when no external field exists [13]. This encourages $\mathrm{M}$. Dirar and others to propose the existence of a vacuum potential to cure this defect [13].

The study made by M. Lodari, et al. shows that the effective mass of holes increases almost linearly with holes density. However the applied magnetic field does not change the effective mass appreciably. This phenomenon is observed in undoped Ge/SiGe. The effective mass is about $000.05 \mathrm{~m}$ at zero density. These predictions need to be confirmed by experiments [14]. In the paper of R. Khordad the position dependent effective mass changes and increases the total refractive index at low photon energy, while it decreases just after a critical photon energy. The linear and total absorption coefficient increases in the presence of the effective mass compared to the ordinary mass. Unfortunately such results are not confirmed empirically [15]. Microwave photo resistance measurements made by A. T. Htke and others show that the value of the effective mass in high mobility GaAs/AlGaAs quantum wells is about $12 \%$ more than the band mass in GaAs. This indicates existence of electron-electron interaction.

However the magnetoplasma dispersion measurements revealed an effective mass close to the GaAs band mass. Slight decrease in the effective mass is observed upon increasing the magnetic field strength. [16]. In the paper of Geoffroy Hauteir, the oxides that have lower effective mass are $\mathrm{ZnO}$ and $\mathrm{IN}_{2} \mathrm{O}_{3}$. Computer analysis shows that the effective mass is affected by the oxygen hybridization $s$ orbital besides and d orbital characters [17]. The work done by Guog-quang Hai shows that the effective mass is affected by the width of the quantum barrier. The increase of the barrier width decreases both the effective mass and the polaron binding energy after a certain critical value. This is done in the absence of the external magnetic field. This result is in direct conflict with the conventional approach which predicts vanishing of the effective mass in the absence of any external field [18].

To widen the scope of solving the effective mass problem, an elegant model is proposed in this work. First of all the conventional model is rederived using simpler mathematical relations. The quantum laws and Newton laws are used to construct a model which uses the concept of vacuum force, to prove that the effective mass reduced to the ordinary mass without any need of introducing the notion of external force. This is done in Sections 2 and 3. The conclusion is in Section 4 New Derivation of the Old Effective Mass Model.

\section{NEW DERIVATION OF THE CONVENTIONAL EFFECTIVE MASS USING THE ACCELERATION CONCEPT}

The old ordinary expression for the effective mass can be derived here using simple mathematics. According to Newton's second law, one gets 


$$
m \frac{\mathrm{d} v}{\mathrm{~d} t}=F_{e}+F_{i}
$$

where $m, v, F_{e}, F_{i}$ stands for mass, velocity external force, and lattice crystal force respectively. One can use the effective mass $m^{*}$ and wave number $K^{*}$ to get

$$
\frac{\mathrm{d} v}{\mathrm{~d} t}=\frac{\mathrm{d} v}{\mathrm{~d} K^{*}} \frac{\mathrm{d} K^{*}}{\mathrm{~d} t}=\frac{1}{m^{*}} \frac{\mathrm{d} m^{*} v}{\mathrm{~d} K^{*}} \frac{\mathrm{d} K^{*}}{\mathrm{~d} t}
$$

where the momentum $P$ in classical and quantum is given by

$$
P^{*}=m^{*} v=\hbar K^{*}
$$

Hence

$$
\frac{\mathrm{d} m^{*} v}{\mathrm{~d} K^{*}}=\frac{\mathrm{d} \hbar K^{*}}{\mathrm{~d} K^{*}}=\hbar
$$

But according to Newton's sec on law, when the crystal force is hidden inside $K^{*}$, the expected force is only the external one, hence

$$
\frac{\mathrm{d} P^{*}}{\mathrm{~d} t}=\hbar \frac{\mathrm{d} K^{*}}{\mathrm{~d} t}=F_{e}
$$

However when crystal and external force appears explicitly, the second law reads

$$
\hbar \frac{\mathrm{d} K}{\mathrm{~d} t}=F_{e}+F_{i}
$$

Thus using Equations (2), (4) and (5) gives:

$$
\frac{\mathrm{d} v}{\mathrm{~d} t}=\frac{1}{m^{*}} \hbar \frac{F_{e}}{\hbar}=\frac{F_{e}}{m^{*}}
$$

Multiplying both sides by $m$, and using Equation (1) gives

$$
m \frac{\mathrm{d} v}{\mathrm{~d} t}=\frac{m}{m^{*}} F_{e}=F_{e}+F_{i}
$$

Thus

$$
m^{*}=\frac{m F_{e}}{F_{e}+F_{i}}
$$

which is the conventional expression for the effective mass $\mathrm{m}^{*}$.

Another very simple approach based on classical laws can be performed. Using again Newton's Second Law of Motion, for acceleration a, mass $\mathrm{m}^{*}$, and external force $F_{e}$, one gets

$$
m^{*} a=F_{e}
$$

where the crystal force is hidden in the mass $m^{*}$ and does not appear explicitly. However it appears explicitly in the form

$$
m a=F_{e}+F_{i}
$$

In view of Equations (10) and (11), one gets

$$
\frac{m^{*} a}{m a}=\frac{F_{e}}{F_{e}+F_{i}}
$$

Cancelling the acceleration on the left hand side gives 


$$
\begin{aligned}
\frac{m^{*}}{m} & =\frac{F_{e}}{F_{e}+F_{i}} \\
m^{*} & =\frac{m F_{e}}{F_{e}+F_{i}}
\end{aligned}
$$

which is again the ordinary effective mass relation when no crystal field exists, i.e.

When

$$
\begin{aligned}
& F_{i}=0 \\
& m^{*}=m
\end{aligned}
$$

Thus effective mass is equal to the ordinary mass, which can form with experiments. However when the crystal field exists and when no external force exists, i.e. when

$$
\begin{aligned}
& F_{e}=0 \\
& m^{*}=0
\end{aligned}
$$

which is in direct conflict with observations. This is since the electron mass or the mass of any elementary particle does not vanish in the absence of external forces. This means that one needs to modify this relation to cure this defect.

\section{VACUUM EFFECTIVE MASS MODEL}

The defect of the expression of the effective mass can be cured with the aid of the concept of vacuum energy. The existence of vacuum energy, i.e., Casimir effect, is the well known phenomena which confirm experimentally the existence of vacuum force and energy. According to this version the total force acting on the electron is the vacuum force $F_{v}$, beside $F_{e}$ and $F_{i}$, thus one gets

$$
m \frac{\mathrm{d} v}{\mathrm{~d} t}=F_{e}+F_{i}+F_{v}
$$

But the left hand side of Equation (18) can be rewritten in terms of $K^{*}$ to be

$$
m \frac{\mathrm{d} v}{\mathrm{~d} t}=m \frac{\mathrm{d} v}{\mathrm{~d} K^{*}} \frac{\mathrm{d} K^{*}}{\mathrm{~d} t}
$$

Using Equations (2), (3) and (4) gives

$$
\frac{\mathrm{d} v}{\mathrm{~d} K^{*}}=\frac{1}{m^{*}} \frac{\mathrm{d} m^{*} v}{\mathrm{~d} K^{*}}=\frac{1}{m^{*}} \frac{\mathrm{d} \hbar K^{*}}{\mathrm{~d} K^{*}}=\frac{\hbar}{m^{*}}
$$

With the aid of Equation (5), inserting $F_{v}$, gives

$$
\frac{\mathrm{d} P^{*}}{\mathrm{~d} t}=\frac{\hbar \mathrm{d} k^{*}}{\mathrm{~d} t}
$$

Thus (21) gives

$$
\frac{\mathrm{d} K^{*}}{\mathrm{~d} t}=\frac{F_{e}+F_{v}}{\hbar}
$$

Inserting (20) and (22) in (19) gives

$$
m \frac{\mathrm{d} v}{\mathrm{~d} t}=m \frac{\hbar}{m^{*}} \frac{F_{e}+F_{v}}{\hbar}=\frac{m}{m^{*}}\left(F_{e}+F_{v}\right)
$$

Using Equations (18) and (23) gives 


$$
F_{e}+F_{v}+F_{i}=\frac{m}{m^{*}}\left(F_{e}+F_{v}\right)
$$

Therefore

$$
m^{*}=\frac{m\left(F_{e}+F_{v}\right)}{F_{e}+F_{i}+F_{v}}
$$

When no crystal force exists

$$
F_{i}=0
$$

Thus

$$
m^{*}=m \frac{F_{e}+F_{v}}{F_{e}+F_{v}}=m
$$

which agree with experiments and previous results.

Also when no force exists at all

$$
F_{e}=0, F_{i}=0
$$

Thus according to Equation (26)

$$
m^{*}=\frac{m F_{v}}{F_{v}}=m
$$

which agrees with observations and does not agree with the result predicted by the conventional model.

The same new equation can be also directly obtained by using directly Newton's second laws without any need for quantum laws, where

$$
m^{*} a=F_{e}+F_{v}
$$

Again the crystal force is hidden in $m^{*}$, while $F_{e}$ and $F_{v}$ appears explicitly. When all forces appear explicitly, one gets

$$
m^{*} a=F_{e}+F_{i}+F_{v}
$$

Dividing (29) by (30) gives

$$
\frac{m^{*}}{m}=\frac{m^{*} a}{m a}=\frac{F_{e}+F_{v}}{F_{e}+F_{i}+F_{v}}
$$

Thus the effective mass takes the form

$$
m^{*}=\frac{m\left(F_{e}+F_{v}\right)}{F_{e}+F_{i}+F_{v}}
$$

Vacuum energy is small, one can ignore it to get, the old expression

$$
m^{*}=\frac{m F_{e}}{F_{e}+F_{i}}
$$

The effect of crystal force can be obtained without any need to incorporate the external force, which does not give correct value of the effective mass which is dependent strongly on the external force in the old model. Concerning the crystal and vacuum force only, Newton's second law reads

$$
m \frac{\mathrm{d} v}{\mathrm{~d} t}=F_{e}+F_{i}
$$

Using the same procedures as in Equation (2), one gets 


$$
m \frac{\mathrm{d} v}{\mathrm{~d} t}=m \frac{\mathrm{d} v}{\mathrm{~d} K^{*}} \frac{\mathrm{d} K^{*}}{\mathrm{~d} t}
$$

Using the momentum definition (3) gives

$$
\frac{\mathrm{d} v}{\mathrm{~d} K^{*}}=\frac{1}{m^{*}} \frac{\mathrm{d} m^{*} v}{\mathrm{~d} K^{*}}=\frac{1}{m^{*}} \hbar \frac{\mathrm{d} K^{*}}{\mathrm{~d} K^{*}}=\frac{\hbar}{m^{*}}
$$

But when the crystal force is hidden one gets:

$$
\hbar \frac{\mathrm{d} K^{*}}{\mathrm{~d} t}=F_{v}
$$

Thus

$$
\frac{\mathrm{d} K^{*}}{\mathrm{~d} t}=\frac{F_{v}}{\hbar}
$$

Inserting Equations (36) and (37) in (35) yields

$$
m \frac{\mathrm{d} v}{\mathrm{~d} t}=m \frac{\hbar}{m^{*}} \frac{F_{v}}{\hbar}=\frac{m}{m^{*}} F_{v}
$$

Comparing (34) and (38) gives

$$
F_{v}+F_{i}=\frac{m}{m^{*}} F_{v}
$$

Hence the effective mass is given by

$$
m^{*}=\frac{F_{v} m}{F_{v}+F_{i}}
$$

When no crystal force exists

$$
F_{i}=0
$$

Thus

$$
m^{*}=m \frac{F_{v}}{F_{v}}=m
$$

which agrees with experiments similarly one can use Equation (29) by ignoring $F_{e}$, to get

$$
m^{*} a=F_{v}
$$

Also using Equation (30) and ignoring $F_{e}$, yields

$$
m a=F_{v}+F_{i}
$$

Using Equations (34) and (44), yields

$$
\frac{m^{*}}{m}=\frac{m^{*} a}{m a}=\frac{F_{v}}{F_{v}+F_{i}}
$$

Again the effective mass is given by

$$
m^{*}=\frac{m F_{v}}{F_{v}+F_{i}}
$$

This expression can also be derived using Psr (gsr) expression for the mass [12]

$$
m=\frac{m_{o}}{\left(1+\frac{2 \varphi}{c^{2}}-\frac{v^{2}}{c^{2}}\right)^{\frac{1}{2}}}
$$


For very slow velocity, such that

$$
\begin{gathered}
v<\varphi \ll c \\
\left(1+\frac{2 \varphi}{c^{2}}-\frac{v^{2}}{c^{2}}\right)^{\frac{1}{2}}=\left(1+\frac{2 \varphi}{c^{2}}\right)^{\frac{1}{2}}=1+\frac{\varphi}{c^{2}}=\frac{m_{o} c^{2}+m_{o} \varphi}{m_{o} c^{2}}=\frac{V_{v}+V}{V_{v}} \\
=\frac{\left(V_{v}+V\right) L}{V_{v} L}=\frac{F_{v}+F_{i}}{F_{v}}
\end{gathered}
$$

where one assumes that the zero point energy stands for the vacuum energy. For uniform fields inside cubic crystal of dimension $L$

$$
\begin{gathered}
V_{v}=F_{v} L \\
V=F_{i} L
\end{gathered}
$$

Thus, since

$$
m^{*}=m, m=m_{o}
$$

It follows that Equation (47) gives

$$
m^{*}=\frac{m F_{v}}{F_{v}+F_{i}}
$$

When no crystal field exists

$$
m^{*}=m
$$

\section{DISCUSSION}

Using the Newtonian and quantum expressions of momentum and its relation with the force (1 - 5), a new simple derivation has been used to derive the conventional expression of the effective mass (9). Newton's second law of motion (11) and (12) only has also been used to find relation (13). When no external field exists (see (16)), the effective mass vanishes (see (7)). This does not agree with observations as far as the effective mass reduces to the ordinary mass in this situation.

To cure this defect one incorporates vacuum force to both Equations (18) and (21). In Equation (18) the external crystal and vacuum forces appear explicitly, whereas the crystal force does not appear explicitly in Equation (21) to be impeded in the effective mass. The effective mass obtained, using Newton's and quantum laws, includes new vacuum force as compared to the ordinary one as shown by Equation (24). Using Newton's second law only atypical expression has been obtained also as Equation (31) shows. The two expressions reduced to the conventional one (13) in the absence of vacuum force. Unlike the conventional model when no forces exist the effective mass equals the ordinary mass as Equation (28) indicates.

To account for the effect of the crystal force a novel model has been proposed using vacuum force and removing the external force (see (40), (46), (51)), using Newton's, quantum and Psr (gsr) laws. In the absence of crystal field the effective mass reduced to the ordinary mass as shown by Equations (42) and (52).

\section{CONCLUSION}

Classical Newton's second law alone or combined with the expression of quantum momentum beside Psr (gsr) has been used to find a new novel expression for the crystal effective mass. This expression accounts for the vacuum Casimir force and reduces to the ordinary mass in the absence of forces. Thus it 
cures the defect of the conventional model in which the mass vanishes in the absence of the external force.

\section{CONFLICTS OF INTEREST}

The authors declare no conflicts of interest regarding the publication of this paper.

\section{REFERENCES}

1. Ibach, H. and Luth, H. (2009) Solid State Physics: An Introduction to Principles of Materials Science. Springer, Berlin. https://doi.org/10.1007/978-3-540-93804-0

2. Mehta, N. (2011) Applied Physics for Engineers. PHI Learning Private Limited, New Delhi.

3. Siddalingappa, B. (2010) Engineering Physics. Himalaya Publishing House, Mumbai.

4. Hook, J.R. and Hall, H.E. (1991) Solid State Physics (Manchester Physics Series). Wiley, Hoboken.

5. Wassif, R.K. (1998) Theory of Solids. Universities Publishing House, Cairo.

6. Kittle, C. (1976) Introduction to Solid State Physics. John Wiley and Sons, New York.

7. Derck, F.L. (1982) An Introduction to Tensor Calculus and Relativity. John Wiley and Sons, New York.

8. Ahang, Y.Z. (1998) Special Relativity and Its Experimental Foundations. World Scientific, Singapore.

9. Beser, A. (2002) Concept of Modern Physics. McGraw Hill, London.

10. Dirar, M., Hillo, M.H.M., Abd Elgani, R. and Bakheet, A.M.A. (2013) Neutrino Speed Can Exceed the Speed of Light within the Framework of the Generalized Special Relativity and Savckas Model. Natural Science, 5, 685-688. https://doi.org/10.4236/ns.2013.56084

11. Hillo, M.H.M., Abd Elgani, R., Abd Elhai, R., Abd Allah, M.D. and Elfaki, A.A.A. (2014) Deriving of Generalized Special Relativity (GSR) by Using Mirror Clock and Lorentz Transformations. Natural Science, 6, 1275-1281.

12. Hillo, M.H.M. (2011) Using the Generalized Special Relativity in Deriving the Equation of the Gravitational Redshift. Journal of Modern Physics, 2, 370-373.

13. Dirar Abdalla, M., et al. (2016) The Effect of Speed and Potential on Time, Mass and Energy on the Basis of Newton and Relativity Prediction. International Journal of Engineering Science and Management, 6, 63-79.

14. Lodari, M.M.M., Tosato, A.A.A., Sabbagh, D., Schubert, M.A., Capellini, G., Smmak, A., Veldhorst, M. and Scappucci, G. (2019) Light Effective Hole Mass in Undoped Ge/SiGe Quantum Wells. Physical Review B, 100, 041304(R). https://doi.org/10.1103/PhysRevB.100.041304

15. Khordad, R. (2011) Effect of Position Dependent Effective Mass on Linear and Non Linear Optical Properties of Cubic Quantum Dot. Physica B, 406, 3911-3916. https://doi.org/10.1016/j.physb.2011.07.022

16. Hatke, A.T., Zudo, M.A., Watson, J.D., Manfra, M.J., Peiffer, L.N. and West, K.W. (2013) Evidence of Effective Mass Reduction in GaAs/AlGaAs Quantum Wells. Physical Review B, 87, 161307(R). https://doi.org/10.1103/PhysRevB.87.161307

17. Hauteir, G., Miglio, A., Waroquiers, D., Rignanese, G.M. and Gonze, X. (2014) How Does Chemistry Influence Electron Effective Mass in Oxides? A High Throughput Computational Analysis. Chemistry of Materials, 26, 5447-5458. https://doi.org/10.1021/cm404079a

18. Hai, G.Q., Peeler, F.M. and Devreese, J.T. (1990) Polaron Energy and Effective Mass in a Quantum Wells. Physical Review B: Condensed Matter, 42, 11063-11072. https://doi.org/10.1103/PhysRevB.42.11063 


\section{NOMENCLATURE}

m: mass

$m^{*}:$ effective mass

$F_{e}$ : external force

$F_{i}$ : lattice internal force

$K$ : wavenumber

$K^{*}$ : effective wave number

$P$ : momentum

$P$ : effective momentum

$t$. time

a: acceleration

$F_{v}$ v vacuum force

$m_{o}$ : rest mass

$\varphi$ : potential per unit mass 